\title{
SOUNDGATE: UM DISPOSITIVO SONORO PARA MEDIR PERÍODOS ${ }^{+*_{1}}$
}

Edy Wilson Ferreira Mendes da Silva

Shirley Takeco Gobara

Departamento de Física - UFMS

Campo Grande - MS

\begin{abstract}
Resumo
Neste artigo, relatamos a aplicação pedagógica e a construção de um dispositivo experimental de baixo custo para medir periodos, em particular, de um pêndulo simples. $O$ baixo custo se refere a instrumentos que se encontram à disposição dos professores nas escolas. Seu funcionamento é similar ao Photogate, um dispositivo eletrônico com um emissor e um receptor de luz infravermelha. $O$ novo dispositivo proposto funciona usando o som no lugar da luz, por isso o batizamos com o nome de Soundgate.
\end{abstract}

Palavras-chave: Som; período; experimento; Soundgate.

\begin{abstract}
At the present article we refer to the pedagogical use and the construction of an experimental device whose cost is minimum to measure periods, specifically of a simple pendulum. In this case the low-cost instruments are available to the teachers at schools. Its operation is similar to the Photogate, an electronics device that contains emission and reception of infrared light. The
\end{abstract}

\footnotetext{
${ }^{+}$Soundgate: A sound device to measure time

* Recebido: julho de 2008.

Aceito: maio de 2009.

${ }^{1}$ Apoio FUNDECT
}

Cad. Bras. Ens. Fís., v. 26, n. 2: p. 379-393, ago. 2009. 
new suggested device changes light into sound during its operation so we denominated it, Soundgate.

Keywords: Sound; period; experiment; Soundgate.

\section{Introdução}

O Grupo Interdisciplinar de Pesquisa em Ensino de Ciência (GINPEC) da Universidade Federal do Mato Grosso do Sul desenvolve alguns projetos em Ensino de Ciências na área da Física, com o objetivo de pesquisar processos de ensino e aprendizagem e propor metodologias, objetos e/ou ambientes de aprendizagem, dispositivos experimentais, etc., que trabalhem com os conceitos físicos de ondas, em particular, ondas sonoras. Este artigo trata de um desses projetos e propõe a utilização de um dispositivo experimental, o Soundgate, um instrumento desenvolvido para medir períodos de um pêndulo simples, possibilitando determinar o valor da aceleração gravitacional com uma boa precisão.

\section{O Soundgate}

Com a informatização das escolas, o computador é uma realidade presente no ensino, e muito útil, em especial, em ciência. Um dos desafios do grupo GINPEC é pesquisar e propor formas de usar essa tecnologia para trabalhar os conceitos de ondas sonoras, mas de maneira inovadora. Várias possibilidades estão sendo pesquisadas e, dentre elas, optamos por utilizar o som como ferramenta para o estudo de outro conceito físico: o de movimento periódico.

O Soundgate tem o seu princípio de funcionamento baseado no Photogate, um dispositivo eletrônico com diodo emissor de luz e um detector de luz - uma fotocélula. Essa fotocélula é ativada ou desativada quando um objeto opaco passa em sua frente, interrompendo o sinal luminoso do diodo emissor de luz. No Soundgate, substituímos o diodo emissor de luz por um alto-falante e a fotocélula, por um microfone. Trata-se de um dispositivo que "percebe" quando um objeto passa entre o alto-falante e o microfone, pois a intensidade do som captada pelo microfone diminui.

Esse dispositivo utiliza a placa de som, o microfone e o alto-falante na sua montagem física. Para gerenciá-lo, foi desenvolvido um software utilizando o Macromedia Flash associado a uma página html. O software gerenciador encon- 
tra-se disponível gratuitamente para ser usado diretamente na internet, dispensando assim sua instalação.

\section{II.1 Montagem}

A montagem da parte física é simples e não requer nenhum conhecimento técnico especializado, bastando ligar os alto-falantes e o microfone no computador e calibrá-los.

Um dos alto-falantes deve ser posicionado de frente para o microfone, a uma distância de $5 \mathrm{~cm}$ a $10 \mathrm{~cm}$, conforme mostra a Fig. 1.

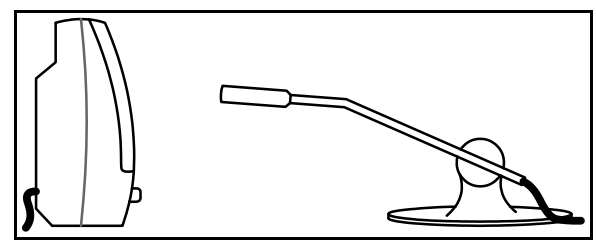

Fig. 1

\section{II.2 Calibragem}

O Soundgate encontra-se hospedado no endereço:

$<$ http://www.edy.pro.br/Soundgate>.

Após acessar o endereço, o computador abrirá automaticamente uma caixa de diálogo (Fig. 2) e o usuário deverá clicar na opção "Permitir", para que o Soundgate tenha acesso ao alto-falante e ao microfone.

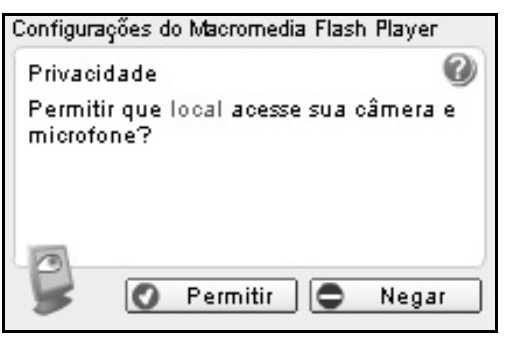

Fig. 2

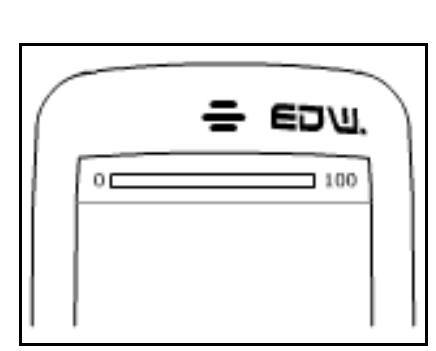

Fig. 3

Com o Soundgate aberto, o microfone e o altofalante posicionados, o usuário deverá abaixar o volume do som e iniciar a cronometragem clicando no botão "Iniciar" do programa e, gradualmente, deverá ir aumentando o volume do som até o sensor do Soundgate atingir o nível 100 (ver no visor do programa - Fig. 3). Depois deverá passar a mão entre o alto-falante e o microfone e verificar se o nível do sensor diminui sensivelmente. Caso isso 
não aconteça, deve-se abaixar o volume e iniciar novamente a calibragem.

Observação: O nível do sensor deve permanecer no máximo quando não houver nada entre o alto-falante e o microfone, e deve cair quando algum obstáculo for colocado entre eles.

\section{Soundgate: Experimentos}

Para a utilização do Soundgate, sugerimos dois experimentos: um para estudar as grandezas físicas que influenciam no período do pêndulo simples e outro para determinar a aceleração local da gravidade.

\section{III.1 Pêndulo Simples}

Essa experiência pode ser trabalhada com alunos do nono ano do Ensino Fundamental ou do primeiro ano do Ensino Médio. Nessa atividade, trabalha-se a dependência do período com as grandezas: massa, comprimento do fio e ângulo de oscilação. Em geral, essa experiência é feita com cronômetros digitais ou analógicos, o que toma tempo, necessitando de toda a aula para a experimentação. Outro fator desfavorável para medir manualmente o período, é que nem sempre os resultados obtidos têm boa precisão. Com o uso do Soundgate, ganha-se em precisão nos dados coletados e no tempo da experimentação. O tempo extra ganho com o uso do Soundgate permite ao professor apresentar o conteúdo, discutir o que será observado no experimento e levar os alunos a levantarem hipóteses relacionadas aos fatores que podem influenciar no período de oscilação do pêndulo, para depois verificar se essas hipóteses se confirmam ou não durante o experimento.

No anexo 1, encontra-se uma sugestão de roteiro para a realização dessa experiência.

\section{III.2 Aceleração Gravitacional}

Determinar o valor local da aceleração da gravidade é uma atividade interessante para alunos do Ensino Médio, mas não é adequada para o Ensino Fundamental, porque é necessário que os estudantes tenham conhecimento de alguns conceitos físicos mais específicos e um maior rigor matemático que, usualmente, não são desenvolvidos no nível Fundamental. 


\section{III.3 Montagem do Pêndulo}

Para a construção do pêndulo serão necessários:

- Linha de algodão $\mathrm{n}^{0} 10$.

- Madeira (Retalho de madeira ou de MDF).

- Suporte para o pêndulo.

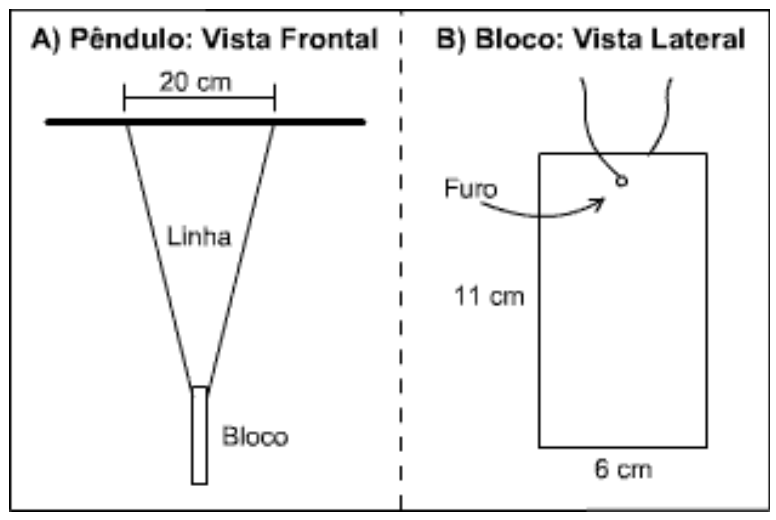

Fig. 4

Para a utilização do Soundgate, deve-se montar pêndulos de acordo com o esquema da Fig. 4-A e confeccionar blocos de madeira, conforme Fig. 4-B.

Primeiramente, para a confecção do pêndulo, deve-se cortar um bloco de madeira ou de MDF no tamanho aproximado de $11 \mathrm{~cm} \times 6 \mathrm{~cm}$, com a espessura variando de $1 \mathrm{~cm}$ a $1,5 \mathrm{~cm}$ e fazer um furo em uma das extremidades do bloco, como mostra a Fig. 4-B. Essa talvez seja a parte mais trabalhosa para o professor.

Para verificar a dependência do período de oscilação do pêndulo com a massa, é necessário confeccionar blocos de mesma área, mantendo as mesmas dimensões para a largura e altura $(11 \mathrm{~cm}$ x $6 \mathrm{~cm})$, porém com espessuras diferentes.

Para manter o movimento do pêndulo unidimensional, a linha deve apenas atravessar o furo do bloco (sem amarrar). As pontas livres da linha devem ser fixadas no suporte a uma distância aproximada de $20 \mathrm{~cm}$ de uma ponta a outra, conforme Fig. 4-A.

Observação: Como suporte para o pêndulo, use hastes rígidas ou cabos de vassouras apoiados sobre mesas e/ou cadeiras. 


\section{Ao professor}

O Soundgate foi projetado para registrar o instante em que o objeto passa entre o alto-falante e o microfone e não para registrar o período. Assim, os alunos devem determinar o período de oscilação do pêndulo, calculando o intervalo de tempo entre dois instantes de índice par.

O primeiro tempo (instante) marcado pelo Soundgate deve ser descartado, pois esse primeiro instante depende de variáveis que podem influenciar no valor correto, como: o momento em que o aluno coloca o pêndulo para oscilar e o instante em que o Soundgate é ligado; os demais instantes dependem somente do período de oscilação do pêndulo.

Para iniciar as atividades, é interessante que o professor discuta o conceito de centro de massa para que o aluno entenda como se efetua a medida do comprimento do pêndulo, ou seja, a distância do suporte ao centro de massa do bloco. Para determinar o centro de massa dos blocos, basta encontrar a intersecção das diagonais da superfície retangular deles, visto que, no experimento, são utilizados blocos retangulares.

Na primeira sugestão de experimento (Roteiro I - Pêndulo Simples), o período de oscilação do pêndulo depende somente da aceleração local da gravidade e de seu comprimento, mas sugere-se verificar a "influência" da massa e do ângulo no período de oscilação do pêndulo.

A segunda sugestão de atividade (Roteiro II - Usando um pêndulo simples para determinar o valor local da gravidade) tem como objetivo determinar a aceleração da gravidade local e analisar a influência da gravidade no período de oscilação do pêndulo. Como não é possível variar a aceleração local da gravidade, foi proposto um problema a partir de uma situação hipotética: a realização do experimento na Lua. Para verificar o valor da aceleração gravitacional obtida experimentalmente, sugere-se que o professor utilize o valor pré-determinado no artigo "Variação da aceleração da gravidade com a latitude e altitude" (LOPES, 2008).

Para finalizar as atividades, sugere-se que o professor explore os fatores históricos que envolvem o estudo do movimento pendular por meio da leitura do artigo "Metodologia e política em ciência: o destino da proposta de Huygens de 1673 para adoção do pêndulo de segundos como um padrão internacional de comprimento e algumas sugestões educacionais" (MATTHEWS, 2001). 


\section{Considerações finais}

O Soundgate foi desenvolvido, a partir do desafio de usar conceitos físicos sobre onda sonora, para criar um instrumento capaz de medir tempo que tivesse uma aplicação prática.

A proposta de utilização desse dispositivo - o Soundgate -, inicialmente desenvolvido para realizar experimentos sobre o movimento pendular, parte agora para uma segunda etapa: a sua aplicação em experimentos de cinemática, mais especificamente, no estudo dos conceitos de velocidade e aceleração.

O Soundgate não foi desenvolvido com o intuito de substituir o cronômetro e muito menos o Photogate. Trata-se apenas de mais uma opção para o professor realizar atividades experimentais para o ensino de Física.

\section{Bibliografia}

HALLIDAY D.; RESNICK R.; WALKER , J. Fundamentos de Física. 3. ed. Rio de Janeiro: LTC Editora, 1998. v. 1.

BRITO, A. A. S. Um pêndulo simples barato. Revista de Ensino de Física, v. 1, n. 1, p. 14-18, jan. 1979.

BAZIN, M.; LUCIE, P. Por que e como estudar o pêndulo simples no ensino básico? Revista de Ensino de Física, v. 3, n. 1, mar. 1981.

GREF Física 2: Física térmica/Óptica. São Paulo: EDUSP, 1991.

LOPES, W. Variação da aceleração da gravidade com a latitude e altitude. Caderno. Brasileiro de Ensino de Física, v. 25, n. 3, p. 561-568, dez. 2008. Disponível

em:

$<$ http://www.periodicos.ufsc.br/index.php/fisica/article/viewFile/9106/8450>.

Acesso em: 22 abr. 2009.

MATTHEWS, M. Metodologia e política em ciência: o destino da proposta de Huygens de 1673 para adoção do pêndulo de segundos como um padrão internacional de comprimento e algumas sugestões educacionais. Caderno. Brasileiro de Ensino de Física, v. 18, n. 1, p. 7-25, abr. 2001. Disponível em:

$<$ http://www.periodicos.ufsc.br/index.php/fisica/article/view/7084/6555>. Acesso em: 22 abr. 2009. 


\section{Roteiro I: Pêndulo simples}

\section{Resumo}

Um pêndulo simples é um sistema mecânico formado por um corpo ideal que consiste de uma partícula suspensa por um fio inextensível e de massa desprezível. O corpo descreve um arco de circunferência em torno do ponto de oscilação.

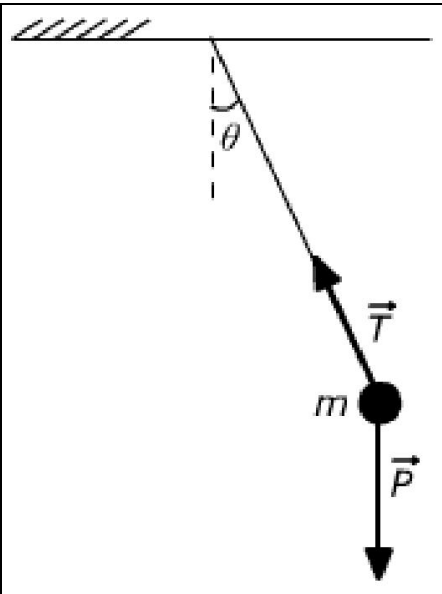

Fig. I-A A descoberta da periodicidade do movimento do pêndulo foi feita por Galileu Galilei (15641642). O movimento de um pêndulo simples envolve basicamente uma grandeza chamada período (simbolizada por T), que é o intervalo de tempo gasto pelo objeto para percorrer toda a trajetória (ou seja, retornar a sua posição inicial, correspondendo a um ciclo do movimento). Associada a essa grandeza, tem-se a frequência (f), definida como a quantidade de ciclos realizados em uma unidade de tempo. Numericamente, a frequência é igual ao inverso do período $(f=1 / T)$. A unidade da frequência no Sistema Internacional de Unidades (SI) é o hertz $(\mathrm{Hz}): 1 \mathrm{~Hz}$ equivale a um ciclo por segundo.

\section{Problemas propostos}

Verificar experimentalmente se o período de um pêndulo simples depende:

- do ângulo de soltura $(\theta)$.

- do comprimento do fio (L).

- da massa (m).

\section{Material}

- Computador com saída de áudio e entrada para microfone.

- Alto-falante.

- Microfone.

-Bases e suportes.

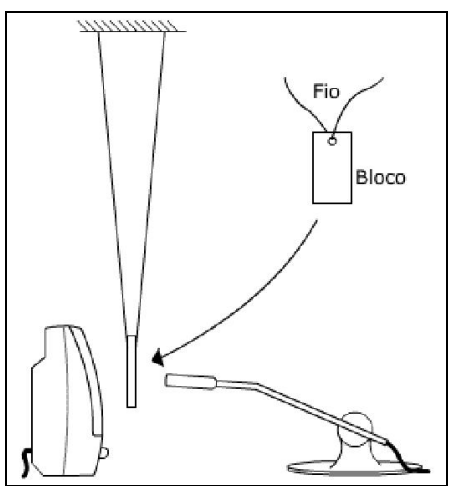

Fig. I-B 
- Um pêndulo simples composto por um fio e um bloco de madeira.

\section{Montagem do experimento}

Monte o experimento conforme a Fig. I-B e, para maior facilidade de manuseio e cálculo, use um bloco de madeira de forma regular e retangular (facilita encontrar o centro de massa) com um furo em uma das extremidades para passar um fio.

\section{Procedimento experimental}

Verifique a montagem do Soundgate, conforme mostra a Fig. I-B e realize as medidas.

Parte 1 - Para verificar a dependência do período com o ângulo de soltura:

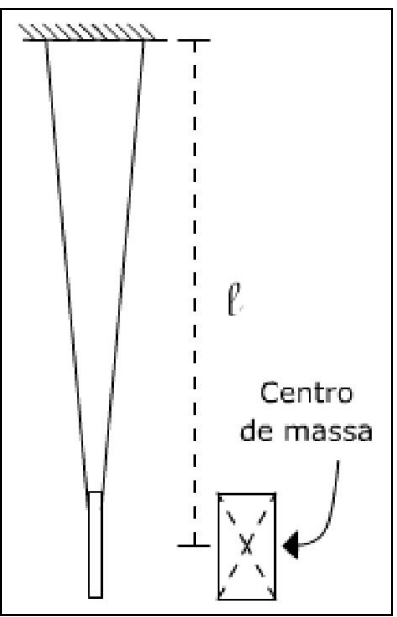

Fig. I-C

1.1 Monte um pêndulo com mais ou menos $75 \mathrm{~cm}$ de comprimento. $\mathrm{O}$ comprimento deve ser medido da base ao centro de massa do bloco; ver Fig. I-C.

1.2 Usando um transferidor para medir o ângulo de oscilação, solte o pêndulo com um ângulo de aproximadamente $5^{\circ}$, anote na Tabela 1-A os doze primeiros valores cronometrados no Soundgate e termine de preencher a Tabela.

1.3 Repita o passo 1.2, mas, agora, usando um ângulo de aproximadamente $10^{\circ}$. Anote os resultados na Tabela 1-B e termine de preencher a Tabela.

Parte 2 - Para verificar a dependência do período com o comprimento do fio:

2.1 Ainda utilizando o mesmo pêndulo da $1^{\mathrm{a}}$ parte do experimento, coloque-o para oscilar. Anote na Tabela 2-A os doze primeiros valores cronometrados no Soundgate e termine de preencher a Tabela. 
2.2 Altere o comprimento do fio para aproximadamente $150 \mathrm{~cm}$ e coloque o pêndulo para oscilar. Anote na Tabela 2-B os doze primeiros valores cronometrados no Soundgate e termine de preencher a Tabela.

Parte 3 - Para verificar a dependência do período com a massa:

3.1 Utilizando ainda o mesmo pêndulo do item 2.2, coloque-o para oscilar. Anote na Tabela 3-A os doze primeiros valores cronometrados no Soundgate e termine de preencher a Tabela.

3.2 Monte novamente o pêndulo com o mesmo comprimento do pêndulo usado no item 3.1, mas, agora, com uma massa diferente e coloque-o para oscilar. Anote na Tabela 3-B os doze primeiros valores cronometrados no Soundgate e termine de preencher a Tabela.

Tabela 1 - Observando a dependência do período com o ângulo de oscilação.

Tabela 1-A

Tabela 1-B

\begin{tabular}{|c|c|c|c|c|c|c|c|}
\hline \multicolumn{4}{|c|}{ Ângulo: } & \multicolumn{4}{|c|}{ Ângulo: } \\
\hline \multirow{3}{*}{$\begin{array}{l}01 \\
02\end{array}$} & \multirow[t]{2}{*}{$\mathrm{t}(\mathrm{s})$} & \multirow{2}{*}{\multicolumn{2}{|c|}{ Períodos }} & \multirow{3}{*}{$\begin{array}{l}01 \\
02\end{array}$} & \multirow[t]{3}{*}{$\mathrm{t}(\mathrm{s})$} & \multirow{2}{*}{\multicolumn{2}{|c|}{ Períodos }} \\
\hline & & & & & & & \\
\hline & & \multirow[b]{2}{*}{$\mathrm{T}_{1}(\mathrm{~s})$} & $02-04$ & & & & $02-04$ \\
\hline 03 & & & & 03 & & $\mathrm{~T}_{1}(\mathrm{~s})$ & \\
\hline 04 & & & $04-06$ & 04 & & & $04-06$ \\
\hline 05 & & $\mathrm{~T}_{2}(\mathrm{~s})$ & & 05 & & $\mathrm{~T}_{2}(\mathrm{~s})$ & \\
\hline 06 & & & $06-08$ & 06 & & & $06-08$ \\
\hline 07 & & $\mathrm{~T}_{3}(\mathrm{~s})$ & & 07 & & $\mathrm{~T}_{3}(\mathrm{~s})$ & \\
\hline 08 & & & $08-10$ & 08 & & & $08-10$ \\
\hline 09 & & $\mathrm{~T}_{4}(\mathrm{~s})$ & & 09 & & $\mathrm{~T}_{4}(\mathrm{~s})$ & \\
\hline 10 & & & $10-12$ & 10 & & & $10-12$ \\
\hline 11 & & $\mathrm{~T}_{5}(\mathrm{~s})$ & & 11 & & $\mathrm{~T}_{5}(\mathrm{~s})$ & \\
\hline 12 & & & & 12 & & & \\
\hline $\mathrm{T}=\sum$ & $15=$ & 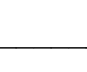 & & $\mathrm{T}=\sum$ & $15=$ & & \\
\hline$f=1 /$ & & & $\mathrm{rtz}$ & $\mathrm{f}=1 /$ & & $\mathrm{H}$ & rtz \\
\hline
\end{tabular}


Tabela 2 - Observando a dependência do período com o comprimento do pêndulo.

Tabela 2-A

\begin{tabular}{|c|c|c|c|}
\hline \multicolumn{4}{|c|}{ Comprimento: } \\
\hline & $\mathrm{t}(\mathrm{s})$ & \multirow{2}{*}{\multicolumn{2}{|c|}{ Períodos }} \\
\hline \multicolumn{2}{|c|}{ Periodos } & & \\
\hline 02 & & \multirow[b]{2}{*}{$\mathrm{T}_{1}(\mathrm{~s})$} & $02-04$ \\
\hline 03 & & & \\
\hline 04 & & \multirow[b]{2}{*}{$\mathrm{T}_{2}(\mathrm{~s})$} & $04-06$ \\
\hline 05 & & & \\
\hline 06 & & \multirow[b]{2}{*}{$\mathrm{T}_{3}(\mathrm{~s})$} & $06-08$ \\
\hline 07 & & & \\
\hline 08 & & \multirow[b]{2}{*}{$\mathrm{T}_{4}(\mathrm{~s})$} & $08-10$ \\
\hline 09 & & & \\
\hline 10 & & \multirow[b]{2}{*}{$\mathrm{T}_{5}(\mathrm{~s})$} & $10-12$ \\
\hline 11 & & & \\
\hline 12 & & & \\
\hline \multicolumn{2}{|c|}{$\mathrm{T}=\sum \mathrm{T}_{\mathrm{n}} / 5=$} & \multicolumn{2}{|c|}{$-s$} \\
\hline \multicolumn{2}{|c|}{$\mathrm{f}=1 / \mathrm{T}=$} & \multicolumn{2}{|c|}{ Hertz } \\
\hline
\end{tabular}

Tabela 2-B

\begin{tabular}{|c|c|c|c|}
\hline \multicolumn{4}{|c|}{ Comprin } \\
\hline & \multirow[t]{2}{*}{$\mathrm{t}(\mathrm{s})$} & \multirow{2}{*}{\multicolumn{2}{|c|}{ Períodos }} \\
\hline 01 & & & \\
\hline 02 & & & $02-04$ \\
\hline 03 & & $\mathrm{~T}_{1}(\mathrm{~s})$ & \\
\hline 04 & & & $04-06$ \\
\hline 05 & & $\mathrm{~T}_{2}(\mathrm{~s})$ & \\
\hline 06 & & & $06-08$ \\
\hline 07 & & $\mathrm{~T}_{3}(\mathrm{~s})$ & \\
\hline 08 & & & $08-10$ \\
\hline 09 & & $\mathrm{~T}_{4}(\mathrm{~s})$ & \\
\hline 10 & & & $10-12$ \\
\hline 11 & & $\mathrm{~T}_{5}(\mathrm{~s})$ & \\
\hline 12 & & & \\
\hline $\mathrm{T}=$ & $15=$ & . & S \\
\hline $\mathrm{f}=1$ & & - H & \\
\hline
\end{tabular}


Tabela 3 - Observando a dependência do período com a massa do pêndulo.

Tabela 3-A

\begin{tabular}{|c|c|c|c|}
\hline \multicolumn{4}{|c|}{ Massa: } \\
\hline & $\mathrm{t}(\mathrm{s})$ & \\
\hline \multicolumn{2}{|c|}{ Periodos } & & \\
\hline 02 & & \multirow[b]{2}{*}{$\mathrm{T}_{1}(\mathrm{~s})$} & $02-04$ \\
\hline 03 & & & \\
\hline 04 & & \multirow[b]{2}{*}{$\mathrm{T}_{2}(\mathrm{~s})$} & $04-06$ \\
\hline 05 & & & \\
\hline 06 & & \multirow[b]{2}{*}{$\mathrm{T}_{3}(\mathrm{~s})$} & $06-08$ \\
\hline 07 & & & \\
\hline 08 & & \multirow[b]{2}{*}{$\mathrm{T}_{4}(\mathrm{~s})$} & $08-10$ \\
\hline 09 & & & \\
\hline 10 & & \multirow[b]{2}{*}{$\mathrm{T}_{5}(\mathrm{~s})$} & $10-12$ \\
\hline 11 & & & \\
\hline 12 & & & \\
\hline \multicolumn{2}{|c|}{$\mathrm{T}=\sum \mathrm{T}_{\mathrm{n}} / 5=$} & \multicolumn{2}{|c|}{$\mathrm{s}$} \\
\hline \multicolumn{2}{|c|}{$\mathrm{f}=1 / \mathrm{T}=$} & \multicolumn{2}{|c|}{ Hertz } \\
\hline
\end{tabular}

Tabela 3-B

\begin{tabular}{|c|c|c|c|}
\hline \multicolumn{2}{|c|}{ Massa: } & & \\
\hline 01 & \multirow{2}{*}{$\mathrm{t}(\mathrm{s})$} & \multicolumn{2}{|c|}{ Períodos } \\
\hline 02 & & & $02-04$ \\
\hline 03 & & $\mathrm{~T}_{1}(\mathrm{~s})$ & \\
\hline 04 & & & $04-06$ \\
\hline 05 & & $\mathrm{~T}_{2}(\mathrm{~s})$ & \\
\hline 06 & & & $06-08$ \\
\hline 07 & & $\mathrm{~T}_{3}(\mathrm{~s})$ & \\
\hline 08 & & & $08-10$ \\
\hline 09 & & $\mathrm{~T}_{4}(\mathrm{~s})$ & \\
\hline 10 & & & $10-12$ \\
\hline 11 & & $\mathrm{~T}_{5}(\mathrm{~s})$ & \\
\hline 12 & & & \\
\hline $\mathrm{T}=\sum \mathrm{T}$ & $15=$ & s & \\
\hline $\mathrm{f}=1 /{ }^{\prime}$ & & Her & \\
\hline
\end{tabular}

\section{Análise dos resultados obtidos}

Analise os valores das Tabelas e responda às questões abaixo.

1. O período de um pêndulo simples depende do ângulo de soltura?

2. O período de um pêndulo simples depende do comprimento do fio?

3. O período de um pêndulo simples depende da massa?

4. Um de seus amigos pede ajuda para arrumar um relógio de pêndulo que está sempre adiantado. Para que o relógio marque corretamente as horas, você deve dizer a seu amigo que:
(01) diminua a massa do pêndulo.
(02) aumente a massa do pêndulo.
(04) diminua o comprimento do fio.
(08) aumente o comprimento do fio.
(16) diminua o ângulo de oscilação.
(32) aumente o ângulo de oscilação. 
Justifique sua resposta.

\section{Roteiro II: Usando um pêndulo simples para determinar o valor local da gravidade}

\section{Resumo}

Isaac Newton foi o primeiro a observar que "No universo tudo se passa como se a matéria atraísse a matéria, na razão direta de sua massa e na razão inversa do quadrado de sua distância". Baseado no estudo sobre a interação entre massas ele formulou o princípio que ficou conhecido como Lei da Atração Gravitacional.

Galileu, em meados do século XVI, observou que o movimento de um pêndulo nos permite determinar a aceleração gravitacional (g). Isso porque o perío-

do (T) de oscilação de um pêndulo depende apenas de duas grandezas: seu comprimento (L) e a aceleração gravitacional local (g). O período pode ser determinado pela expressão:

$$
T=2 \pi \sqrt{\frac{L}{g}}
$$

Isolando $g$ da expressão acima, obtém-se o valor da aceleração gravitacional:

$$
g=4 \pi^{2} \frac{L}{T^{2}} .
$$

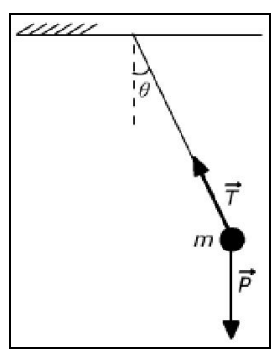

Fig. II. A

\section{Problemas propostos}

Determinar o valor local da gravidade.

\section{Material}

- Computador com saída de áudio e entrada para microfone.

- Alto-falante.

- Microfone.

- Um pêndulo simples composto por um fio e um bloco de madeira.

- Bases e suportes. 


\section{Montagem do experimento}

Monte o experimento conforme a Fig. II-B e, para maior facilidade de manuseio e cálculo, use um bloco de madeira de forma regular e retangular (facilita encontrar o centro de massa) com um furo em uma das extremidades para passar um fio.

\section{Procedimento experimental}

1. Verifique a montagem do Soundgate, conforme a Fig. II-B.

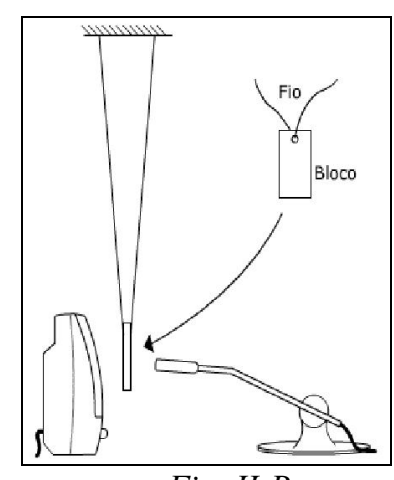

Fig. II-B

2. Marque o centro de massa do bloco de madeira (ver Fig. II-C) para facilitar a medida do comprimento do pêndulo.

3. Usando um transferidor para medir o ângulo de oscilação, afaste o pêndulo da posição de equilíbrio e coloque-o para oscilar a partir de um ângulo inferior a $10^{\circ}$.

4. Anote na Tabela II.1 os primeiros 12 instantes registrados no Soundgate, calcule os 5 primeiros períodos do pêndulo e termine de preencher a Tabela II.1.

5. Usando os valores da Tabela 1, calcule os períodos correspondentes e determine o valor local da gravidade usando a expressão:

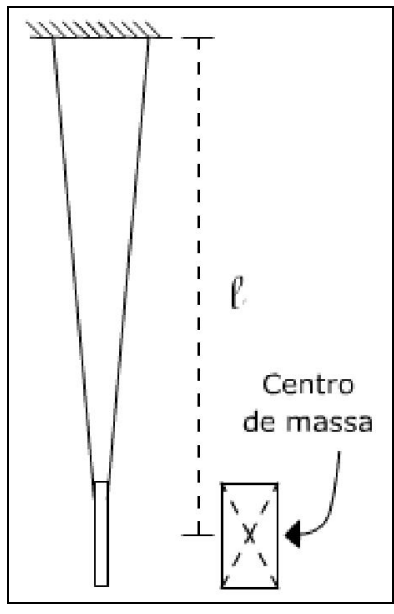

Fig. II-C

$$
g=4 \pi^{2} \frac{L}{T^{2}} .
$$


Tabela II.1

\begin{tabular}{|c|c|c|c|}
\hline \multicolumn{4}{|c|}{ Ângulo: } \\
\hline & $\mathrm{t}(\mathrm{s})$ & \multirow{2}{*}{\multicolumn{2}{|c|}{ Períodos }} \\
\hline 01 & & & \\
\hline 02 & & \multirow[b]{2}{*}{$\mathrm{T}_{1}(\mathrm{~s})$} & $02-04$ \\
\hline 03 & & & \\
\hline 04 & & \multirow[b]{2}{*}{$\mathrm{T}_{2}(\mathrm{~s})$} & $04-06$ \\
\hline 05 & & & \\
\hline 06 & & \multirow[b]{2}{*}{$\mathrm{T}_{3}(\mathrm{~s})$} & $06-08$ \\
\hline 07 & & & \\
\hline 08 & & \multirow[b]{2}{*}{$\mathrm{T}_{4}(\mathrm{~s})$} & $08-10$ \\
\hline 09 & & & \\
\hline 10 & & \multirow[b]{2}{*}{$\mathrm{T}_{5}(\mathrm{~s})$} & $10-12$ \\
\hline 11 & & & \\
\hline 12 & & & \\
\hline \multicolumn{2}{|c|}{$T=\frac{\Sigma T}{5}$} & & \\
\hline & $2 \frac{L}{T^{2}}$ & & \\
\hline
\end{tabular}

\section{Atividade extra}

Sabendo que a aceleração da gravidade na Lua é um sexto da aceleração da gravidade na Terra, se hipoteticamente você montasse esse experimento na Lua, qual seria o período do pêndulo registrado pelo Soundgate na Lua? 